\title{
Density evolution of in-falling prominence material from the 7 th June 2011 CME
}

\author{
Jack Carlyle $^{1,2}$, David Williams ${ }^{1}$, Lidia van Driel-Gesztelyi ${ }^{1,3,4}$ and \\ Davina Innes ${ }^{2}$ \\ ${ }^{1}$ UCL-Mullard Space Science Laboratory, UK \\ email: j.carlyle@ucl.ac.uk \\ ${ }^{2}$ Max Planck Institute for Solar System Research, Germany \\ ${ }^{3}$ Observatoire de Paris, LESIA, UMR 8109 (CNRS), France \\ ${ }^{4}$ Konkoly Observatory, Budapest, Hungary
}

\begin{abstract}
This work investigates the density of in-falling prominence material following the $7^{t h}$ June 2011 eruption. Both the evolution and the distribution of the density is analysed in five discreet "blobs" of material. The density appears to be remarkably uniform, both spatially within the blobs, and temporally over the course of the descent of each, although a slight concentration of material towards the leading edge is noted in some cases. Online material is available at bit.ly/jackblob
\end{abstract}

\section{Introduction}

The Coronal Mass Ejection (CME) on the $7^{\text {th }}$ June 2011 was one of the largest captured by the Solar Dynamics Observatory Atmospheric Imaging Assembly (SDO/AIA), with a large amount of the ejected matter falling back to the solar surface, suggesting high densities (in order for gravitational forces to overcome the magnetic forces, thought to drive CMEs).

As the ejected prominence material fell, it formed discreet blobs, appearing to undergo the magnetic Rayleigh-Taylor (mRT) instability (Innes et al. 2012). By achieving a quantitative assessment of the in-falling material, the physical conditions for such mRT instabilities may be investigated. This investigation assumes photoionisation is the predominant process by which photons are removed from the line of sight, and uses a temporal-interpolative approach to determine the column density of the cool filament material when in absorption against the disc of the Sun (Williams et al. 2013).

\section{Method}

Using 94, 131, 171, 193 and $211 \AA$ AIA images of a blob and unocculted co-spatial frames 5 minutes prior, optical depth of the blob is determined from the observed difference in intensity. These measures are then used to constrain the parameters of the model developed by Williams et al. (2013) which uses a Levenberg-Marquardt least-squares minimisation algorithm to find the best fit for the two free parameters, column density $N_{H}$ and geometric filling factor $G$, in each pixel. For a more thorough description of the event and method, please refer to Carlyle et al. (2013).

\section{Results and Analysis}

Figure 1 shows maps of column density and $G$ for the first blob analysed. All analysed blobs displayed column densities of approximately $2 \times 10^{19} \mathrm{~cm}^{-2}$, remaining constant over their descent. The value of $G$ is approximately 0.8 (with the edge of the blob defined 

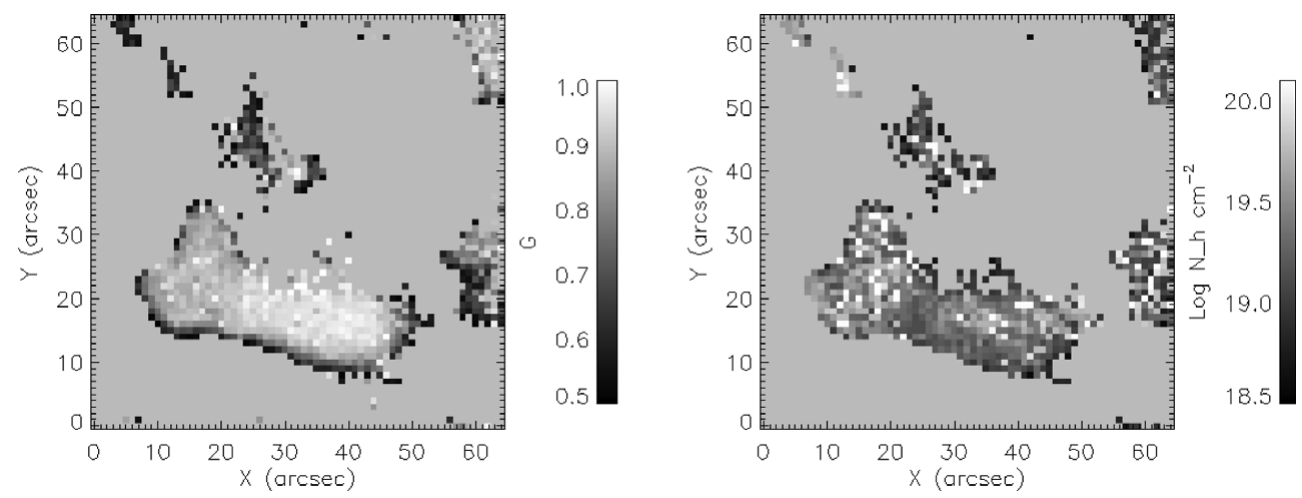

Figure 1. Values obtained for $\mathrm{G}$ (left) and $\mathrm{N}_{H}$ (right) of one blob as it first appeared against the solar disc at 07:06 UT. The direction of travel is approximately $45^{\circ}$ to the negative $\mathrm{x}$-axis.

by $G>0.5$, as beyond this, the majority of emission in the pixel is unattenuated). The constant value of $N_{H}$ suggests the blobs are being held together, with the material possibly carrying with it a bundle of magnetic field. Higher densities are observed towards the leading edge just before the blobs split, suggesting that we may be observing the mRT instability. The fact that $G$ decreases is likely due to a greater proportion of emission being in the foreground as the blobs fall through the solar atmosphere.

\section{Discussion and Conclusion}

This work has found column density values for the discreet blobs of material which fell back to the Sun after the June $7^{\text {th }} 2011 \mathrm{CME}$ and shown that these are relatively high values. Gilbert et al. (2005) calculated the column density of a prominence to be $1.6 \pm$ $1 \times 10^{19} \mathrm{~cm}^{-2}$ before erupting - a value comparable with those calculated for material in this study, which was seen to expand greatly in the initial eruption.

The distribution of the density is consistent with the behaviour of the mRT instability, and the large separation width between successively divided blobs (relative to their length) implies suppression of the higher- $k$ modes of the instability, consistent with the presence of magnetic fields.

\section{Acknowledgements}

JC thanks UCL and MPI for Impact PhD Studentship. The research leading to these results has received funding from EC FP7 under grant agreement No. 284461 (eHEROES). LvDG's work was supported by Hungarian Research grant OTKA K-081421.

\section{References}

Carlyle, J., Williams, D., van Driel-Gesztelyi, L., Innes, D., Hillier, A., Matthews, S., 2013, ApJ, submitted

Gilbert, H., Holzer, T. E., \& MacQueen, R. M., 2005, ApJ, 618, 524

Innes, D. E., Cameron, R. H., Fletcher, L., Inhester, B., \& Solanki, S. K., 2012, A $\& A$ 540, L10

Williams, D. R., Baker, D., \& van Driel-Gesztelyi, L., 2013, ApJ, 764, 165 